\title{
RADIOR, un logiciel d'initiation à l'optimisation de la radioprotection
}

\author{
J.Y. PIPAUD* \\ (Manuscrit reçu le 7 octobre 1994, accepté le 15 décembre 1994)
}

RÉSUMÉ L'optimisation de la radioprotection offre un sujet bien adapté à la réalisation d'un logiciel de formation, avec ses modes d'expression des connaissances variés : animation, étude de cas, questionnaire. La société PRODIDACT vient de réaliser RADIOR, en collaboration scientifique avec le CEPN. L'objectif de ce didacticiel est de faire découvrir et faire rapidement comprendre le raisonnement ALARA.

ABSTRACT The optimisation of radiation protection is a very good theme for a training software, which presents various means of knowledge expression : animation, case study, multiple choice question. PRODIDACT has just produced RADIOR, with the scientific contribution of CEPN. This courseware aims at leading people to discover and understand the ALARA process.

Parce qu'il est de plus en plus répandu et qu'il offre des moyens d'expression multimédia, l'ordinateur est un outil de formation privilégié. Il offre à l'auteur la possibilité de créer des animations qu'il ne pourrait réaliser au tableau noir ou sur transparent. Il apporte à l'utilisateur des fonctionnalités qui renouvellent l'offre de formation : interactivité, rythme d'apprentissage personnalisé, répétitivité.

La société PRODIDACT* a présenté à La Rochelle 1, les 20 et 21 septembre 1994, RADIOR, logiciel d'initiation à l'optimisation de la radioprotection, réalisé avec la collaboration scientifique du CEPN 2. RADIOR s'adresse à tous les acteurs concernés par l'exposition professionnelle aux rayonnements ionisants : concepteurs d'installation, chefs d'installation, responsables de service, formateurs et, bien entendu, travailleurs exposés. La gestion du risque radiologique et la démarche d'optimisation qui en découle impliquent des connaissances de base et la capacité de raisonner dans le cadre d'une procédure. RADIOR propose des modes d'expression adaptés aux différentes étapes : l'animation pour les connaissances de base, l'étude de cas pour la procédure d'optimisation et le questionnaire pour reprendre en détail le raisonnement ALARA.

* PRODIDACT, Espace Wagner, Bât. C, 13858, Aix-en-Provence Cedex 3. Tél : (16) 42.24.30.70. Fax : (16) 42.39.72.96.

1. Journées sur "L'optimisation de la radioprotection des travailleurs dans les domaines électronucléaire, industriel et médical" organisées par la Section de "Protection technique" de la Société française de radioprotection (SFRP) et le Centre d'étude sur l'évaluation de la protection dans le domaine nucléaire (CEPN).

2. Centre d'étude sur l'évaluation de la protection dans le domaine nucléaire, BP 48, 92263 Fontenayaux-Roses Cedex. 
Les animations illustrent le phénomène de la radioactivité, la notion de dose, les unités de mesure, les hypothèses prises sur la relation dose-effet, la gestion du risque résiduel... Des informations plus approfondies peuvent monter à l'écran par simple clic du curseur de la souris sur certains mots ou sur certaines valeurs numériques.

L'étude de cas présente un travail à accomplir sous rayonnements ionisants (une vanne à réparer). On déroule la procédure d'optimisation avec ses nœuds d'analyse : les actions possibles en matière de radioprotection, leurs coûts, les gains en terme de dose... Un tableau de bord basé sur la méthode du "coût raisonnable" aide l'utilisateur à prendre sa décision.

Le mode "questions à choix multiple" propose un exercice en dix questions : après chaque question, l'utilisateur sélectionne, parmi deux réponses proposées, celle qui lui semble correcte. Un commentaire approfondi lui rappelle les clés du raisonnement ALARA.

D'une durée d'utilisation d'environ deux heures, RADIOR contient 4 chapitres, 14 rubriques et 41 animations. Le parcours est un cheminement à choix qui permet à l'utilisateur de naviguer librement à partir du sommaire . Cependant, l'ordre des chapitres, des rubriques et des animations est étudié pour une acquisition progressive des connaissances.

1. Les rayonnements ionisants

- les rayonnements

- la mesure de la radioactivité

- le parcours des rayonnements

- les expositions

- les effets biologiques

- la dosimétrie

- la mesure du risque radiologique

2. La gestion du risque radiologique

- relation doses-effets

- prévention, précaution, risque

- principes de la radioprotection

- optimisation de la radioprotection

- valeurs de l'homme-sievert

3. L'application du principe d'optimisation

- procédure d'optimisation

- cas pratique

4. Testez vos connaissances

Dix questions-clés sur le déroulement de la procédure d'optimisation, avec la possibilité d'imprimer les questions-réponses. 